
São-João. Horticultura Brasileira 25: 533-537.

\title{
Influência da calagem e da adubação fosfatada no acúmulo de nutrientes e crescimento da erva-de-São-João
}

\author{
Cassandro VT do Amarante ${ }^{1,2}$; Paulo Roberto Ernani ${ }^{1,2}$; Alexandra G de Souza ${ }^{1,3}$ \\ ${ }^{1}$ UDESC-CAV, C. postal 281, 88520-000 Lages-SC; ${ }^{2}$ Bolsista Produtividade em Pesquisa CNPq; ${ }^{3}$ Bolsista CAPES; \\ amarante@cav.udesc.br, ernani@cav.udesc.br
}

\section{RESUMO}

A erva-de-São-João (Hypericum perforatum L.), planta medicinal amplamente utilizada no tratamento humano anti-depressivo, tem sido pouco estudada agronomicamente. Avaliou-se os efeitos da calagem e da adubação fosfatada no acúmulo de nutrientes e no crescimento inicial de plantas dessa espécie. $\mathrm{O}$ experimento foi conduzido em Lages, SC, de julho a dezembro de 2003, em casa de vegetação. Foi utilizado o delineamento experimental inteiramente casualizado (fatorial $4 \times 3$ ), correspondente a quatro valores de $\mathrm{pH}$ $(4,1 ; 5,5 ; 6,0$ e 6,5$)$ e três doses de fósforo $\left(0 ; 50\right.$ e $100 \mathrm{mg} \mathrm{kg}^{-1} \mathrm{de}$ solo), com quatro repetições. Foram cultivadas duas plantas por vaso, num Cambissolo Húmico Álico. Avaliaram-se os teores de N, P, K, $\mathrm{Ca}, \mathrm{Mg}, \mathrm{Mn}, \mathrm{Zn}, \mathrm{Cu}$ e Fe no solo e na parte aérea e o rendimento de massa seca da parte aérea e das raízes. A calagem, combinada com a adubação fosfatada, favoreceu o acúmulo de $\mathrm{Ca}, \mathrm{Mg}, \mathrm{K}, \mathrm{N}$ e $\mathrm{P}$ na parte aérea e o crescimento da erva-de-São-João.

Palavras-chave: Hypericum perforatum L., planta medicinal, nutrição de plantas, massa seca.

\section{ABSTRACT}

Influenceof liming and phosphate fertilization on nutrients accumulation and plant growth of St. John's Wort

St. John's Wort (Hypericum perforatum L.) is a medicinal plant widely used for human anti-depressive treatment, despite of being little studied as a cultivated crop. This work was conducted to evaluate nutrients accumulation and initial growth of this plant species, in response to phosphate fertilization and liming. The experiment was carried out in Lages, Brazil, from July to December of 2003, in a greenhouse. The experiment followed a completely randomized factorial design $(4 \times 3)$, corresponding to four $\mathrm{pH}$ values $(4.1 ; 5.5$; 6.0 , and 6.5) and three rates of $\mathrm{P}\left(0 ; 50\right.$, and $100 \mathrm{mg} \mathrm{kg}^{-1}$ of soil), with four replicates. Two plants were cultivated per pot, in an Inceptisol. The contents of $\mathrm{N}, \mathrm{P}, \mathrm{K}, \mathrm{Ca}, \mathrm{Mg}, \mathrm{Mn}, \mathrm{Zn}, \mathrm{Cu}$, and $\mathrm{Fe}$ in the soil and in the plants were obtained, as well as shoot and root dry matter. The combination of the liming and phosphate fertilization promoted the accumulation of $\mathrm{Ca}, \mathrm{Mg}, \mathrm{K}, \mathrm{N}$, and $\mathrm{P}$ in the aerial part and also plant growth of Hypericum perforatum $\mathrm{L}$.

Keywords: Hypericum perforatum L., medicinal plant, plant nutrition, dry matter.

\section{(Recebido para publicação em 9 de maio de 2006; aceito em 8 de novembro de 2007)}

\begin{abstract}
A erva-de-São-João (Hypericum perforatum L.) é planta herbácea e perene que possui propriedade antidepressiva, atribuída à produção do metabólito secundário hipericina (Southwell \& Bourke, 2001). O crescimento e a produção de hipericina são influenciados, dentre outros fatores, pela nutrição das plantas. Baixos níveis de $\mathrm{N}$ no solo aumentam o teor de hipericina em erva-de-São-João. Por outro lado, a elevação da disponibilidade de $\mathrm{N}$ aumenta o desenvolvimento das plantas, diluindo com isso o teor de hipericina na parte aérea (Briskin et al., 2000). A adição de $\mathrm{Ca}, \mathrm{Mg}, \mathrm{K}$ e $\mathrm{P}$ acarretou efeito similar ao $\mathrm{N}$ sobre o crescimento vegetativo, confirmando o efeito benéfico de macronutrientes para o desenvolvimento das plantas (Lieres et al., 1994).

A alta acidez e a baixa disponibilidade de P na maioria dos solos brasileiros representam as maiores restrições ao desenvolvimento das plantas cultivadas.
\end{abstract}

Provavelmente, estes fatores afetam substancialmente o crescimento das plantas de erva-de-São-João e a produção de hipericina.

Quando o pH do solo é muito baixo, diversos fatores afetam negativamente o desenvolvimento vegetal. Além de reduzir a disponibilidade de alguns nutrientes, principalmente $\mathrm{Ca}, \mathrm{Mg}, \mathrm{N}, \mathrm{P}$ e $\mathrm{Mo}$, valores baixos de $\mathrm{pH}$ favorecem o aumento da concentração de $\mathrm{Al}$ na solução do solo (Ernani et al., 1996). Altas concentrações de $\mathrm{Al}$ inibem o crescimento das raízes, com conseqüências negativas na absorção de água e de nutrientes assim como no desenvolvimento das plantas (Taiz \& Zeiger, 2002). Além disso, a redução do sistema radicular afeta a absorção, principalmente de $\mathrm{P}$, já que este nutriente se movimenta no solo por difusão (Ernani et al., 1996, 2000). Quando o $\mathrm{pH}$ atinge valores superiores a $5,4-5,5$, praticamente todo o Al precipita e o Mn diminui a níveis não tóxicos (Ernani \& Almeida, 1986). O aumento do $\mathrm{pH}$ do solo até aproximadamente 6,5 favorece a disponibilidade de nutrientes e o desenvolvimento das plantas (Ernani et al., 1996).

Em solos ácidos, a disponibilidade de $\mathrm{P}$ para as plantas também é prejudicada devido ao favorecimento da adsorção desse nutriente aos óxidos e hidróxidos de Fe e Al. Com isso, a concentração de $\mathrm{P}$ na solução do solo se torna muito baixa, limitando sua absorção pelas plantas (Ernani et al., 2000). O presente trabalho teve por objetivo avaliar o efeito da calagem e do suprimento de $\mathrm{P}$ num solo ácido sobre o acúmulo de nutrientes e a produção de massa seca em erva-de-São-João.

\section{MATERIAL E MÉTODOS}

$\mathrm{O}$ experimento foi realizado em Lages, de julho a dezembro de 2003, em casa de vegetação, no Centro de Ciên- 


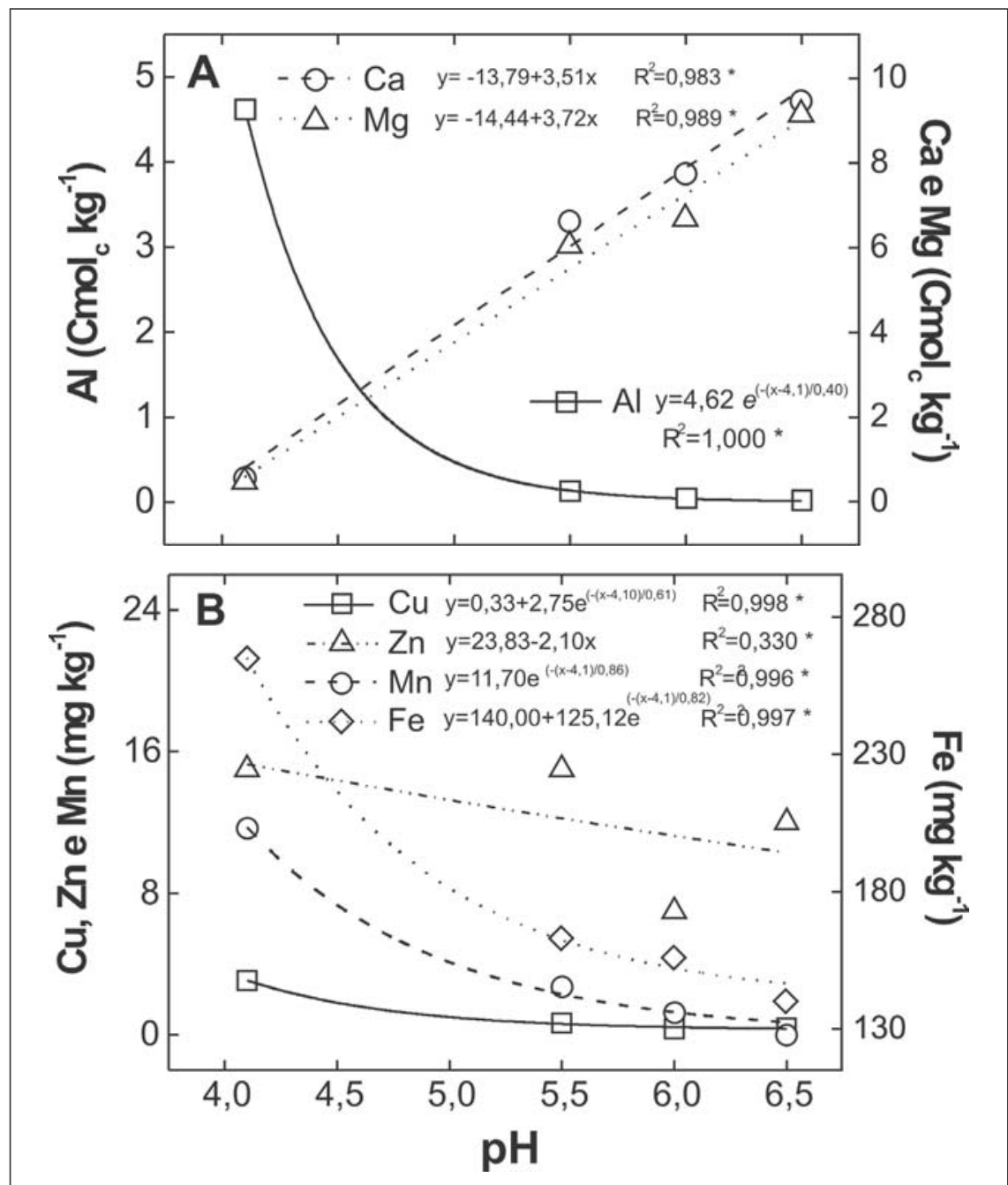

Figura 1. Teores de Al, Ca e Mg (A), Cu, Zn, Mn e Fe (B) no solo em função do aumento do $\mathrm{pH}$ (Contents of $\mathrm{Al}, \mathrm{Ca}$ and $\mathrm{Mg}(\mathrm{A}), \mathrm{Cu}, \mathrm{Zn}, \mathrm{Mn}$ and $\mathrm{Fe}(\mathrm{B})$ in the soil in response to the increment of pH). Lages, UDESC, 2003.

*Significativo a $5 \%$ de probabilidade (significant at $5 \%$ probability).

cias Agroveterinárias. Usou-se um Cambissolo Húmico Álico, coletado em área de campo nativo, na camada de 0 a $20 \mathrm{~cm}$ de profundidade. A análise do solo revelou: $\mathrm{pH}$ em água 4,1 ; índice SMP 4,3; Al trocável $=4,62 \mathrm{cmol} \mathrm{kg}^{-1}$; matéria orgânica $=43 \mathrm{~g} \mathrm{~kg}^{-1}$; argila $=520$ $\mathrm{g} \mathrm{kg}^{-1}$; areia $=290 \mathrm{~g} \mathrm{~kg}^{-1}$; silte $=360 \mathrm{~g} \mathrm{~kg}^{-1}$; $\mathrm{P}$ e K (Mehlich-1) = 4,8 e $144 \mathrm{mg} \mathrm{kg}^{-1}$, respectivamente; e $\mathrm{Ca}$ e $\mathrm{Mg}=0,57$ e 0,48 $\mathrm{cmol}_{\mathrm{c}} \mathrm{kg}^{-1}$, respectivamente.

Foi utilizado o delineamento experimental inteiramente casualizado, em esquema fatorial $4 \times 3$, com quatro valores de $\mathrm{pH}[4,1$ (natural); 5,5; 6,0 e 6,5, correspondendo a $0 ; 7,5 ; 10,5$ e $14,5 \mathrm{~g}$ de calcário dolomítico por kg de solo] e três doses de $\mathrm{P}\left(0,50\right.$ e $100 \mathrm{mg} \mathrm{kg}{ }^{-1}$ de solo). Foram utilizadas quatro repeti- ções, cada qual correspondendo a um vaso com 1,0 kg de solo (base seca) e duas plantas, obtidas por reprodução assexuada, cujas matrizes foram fornecidas pela Estação Experimental de Itajaí da Epagri.

Para elevar o $\mathrm{pH}$ das amostras de solo foi aplicado calcário dolomítico finamente peneirado (preparado em laboratório, com poder relativo de neutralização total PRNT de 100\%), dois meses antes do plantio das mudas, visando permitir a correção do $\mathrm{pH}$ deste tipo de solo para os valores desejados, conforme descrito por Ernani \& Almeida (1986) e Ernani et al. (1996 e 2000). Para suprir as doses de P, foi utilizado fosfato de potássio monobásico $\left(\mathrm{KH}_{2} \mathrm{PO}_{4}\right)$. Devido à presença de $\mathrm{K}$ nes- se fertilizante, seu teor foi equalizado em todos os tratamentos por meio da adição de sulfato de potássio. $\mathrm{O}$ nitrogênio foi aplicado nas unidades experimentais, na dose de $100 \mathrm{mg} \mathrm{kg}^{-1}$ de solo, na forma de uréia, antes do plantio das mudas. No mês de novembro, foram realizadas três aplicações foliares de boro (bórax, 0,1\%) e zinco (sulfato de zinco, $0,1 \%$ ) em todos os tratamentos, visando evitar a deficiência dos mesmos, principalmente nos tratamentos com valores mais elevados de $\mathrm{pH}$. As unidades experimentais receberam água destilada sempre que necessário, por meio da pesagem individual dos vasos.

As plantas foram colhidas manualmente, cinco meses após o plantio das mudas. A parte aérea foi separada do sistema radicular e ambas foram secadas a $60-65^{\circ} \mathrm{C}$, durante $48 \mathrm{~h}$, em estufa com circulação forçada de ar. Foram avaliados os rendimentos de massa seca da parte aérea e do sistema radicular e os teores totais de $\mathrm{N}, \mathrm{P}, \mathrm{K}, \mathrm{Ca}, \mathrm{Mg}, \mathrm{Mn}$, $\mathrm{Zn}, \mathrm{Cu}$ e Fe na parte aérea. As amostras secas da parte aérea foram moídas e digeridas com ácido sulfúrico e água oxigenada num bloco digestor previamente aquecido a $350^{\circ} \mathrm{C}$. As determinações dos nutrientes no tecido vegetal foram realizadas segundo metodologia descrita por Tedesco et al. (1995).

Nas amostras de solo, coletadas após a colheita das plantas, foi determinado o $\mathrm{pH}$ e os teores de N, P, K, Ca, Mg, Al, $\mathrm{Mn}, \mathrm{Zn}, \mathrm{Cu}$ e Fe. $\mathrm{O} \mathrm{pH}$ foi determinado em água, na relação solo:solvente de 1:1. Cálcio, $\mathrm{Mg}, \mathrm{Al}$ e $\mathrm{Mn}$ trocáveis foram extraídos com solução de $\mathrm{KCl} 1 \mathrm{~mol} \mathrm{~L}^{-1}$ e determinados por espectrometria de absorção atômica. $\mathrm{O} \mathrm{K}$ foi extraído com solução ácida (Mehlich-1) e determinado por fotometria de chama. $\mathrm{O} \mathrm{N}$ total foi determinado por destilação com hidróxido de sódio $(\mathrm{NaOH}) 10 \mathrm{~mol} \mathrm{~L}^{-1}$ e posterior titulação com ácido sulfúrico $\left(\mathrm{H}_{2} \mathrm{SO}_{4}\right)$ 0,025 mol L-1. O P foi extraído com solução ácida (Mehlich-1) e determinado por colorimetria, e os micronutrientes $\mathrm{Zn}, \mathrm{Cu}$ e Fe foram extraídos do solo com $\mathrm{HCl} 0,1 \mathrm{~mol} \mathrm{~L}^{-1}$ e determinados por espectrometria de absorção atômica (Tedesco et al., 1995).

Os dados foram submetidos a análises de regressão linear e não linear através dos procedimentos PROC REG e 
PROC NLIN do programa SAS (SAS Institute, Inc.), respectivamente. Os efeitos de níveis de calagem e doses de $\mathrm{P}$ sobre o rendimento total de massa seca da parte aérea e do sistema radicular foram avaliados através de análise de variância e representados através de gráficos em três dimensões, com superfície de resposta ajustada pelo programa Origin 5.1.

\section{RESULTADOS E DISCUSSÃO}

A elevação do pH de 4,1 para valores iguais ou superiores a 5,5 diminuiu

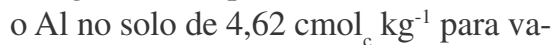
lores muito próximos a zero (Figura 1A). Os teores de $\mathrm{Mn}, \mathrm{Fe}, \mathrm{Cu}$ e $\mathrm{Zn}$ no solo diminuíram com a calagem (Figura 1B), mas permaneceram acima dos valores considerados suficientes (COMISSÃO DE FERTILIDADE DO SOLO - RS/SC, 2004), não tendo provocado sintomas de deficiência nas plantas. No entanto, as plantas apresentaram deficiência de B nos maiores valores de $\mathrm{pH}(6,0$ e 6,5), que foi posteriormente corrigida com aplicações foliares deste micronutriente.

A calagem proporcionou aumento significativo nos teores de $\mathrm{Ca}$ e $\mathrm{Mg}$ no solo, passando, respectivamente, de 0,57 e $0,48 \mathrm{cmol}_{\mathrm{c}} \mathrm{kg}^{-1}$ no $\mathrm{pH} 4,1$, para 9,46 e 9,14 $\mathrm{cmol}_{\mathrm{c}} \mathrm{kg}^{-1}$ no pH 6,5 (Figura 1A). Isto ocasionou incrementos nos teores de Ca e Mg (Figura 2A e B) e na produção de massa seca na parte aérea (Figura 3A). Observou-se que na média dos valores, o teor de Ca na parte aérea passou de 2,62 $\mathrm{mg}$ vaso $^{-1}$ no tratamento sem calagem, para $13,52 \mathrm{mg}$ vaso $^{-1}$ no $\mathrm{pH}$ 6,5 , sendo este incremento maior com a adição de $\mathrm{P}$ ao solo (Figura 2A). No pH 6,5 , o teor de Ca na parte aérea aumentou em aproximadamente $\sim 45 \%$ com a adição de $\mathrm{P}$ (50 e $100 \mathrm{mg} \mathrm{kg}^{-1}$ ) ao solo. $\mathrm{O}$ teor de $\mathrm{Mg}$ na parte aérea, na média dos valores, também aumentou com a calagem, passando de $4,13 \mathrm{mg} \mathrm{vaso}^{-1}$ no $\mathrm{pH} 4,1$ para $25 \mathrm{mg}$ vaso $^{-1}$ no $\mathrm{pH}$ 6,5 (Figura 2B). O maior aumento no teor de $\mathrm{Mg}$ na parte aérea foi verificado na maior dose de $\mathrm{P}$ aplicada, chegando a $32 \mathrm{mg}$ vaso $^{-1}$, no tratamento que tinha $\mathrm{pH}$ 6,5 e dose de $\mathrm{P}$ de $100 \mathrm{mg} \mathrm{kg}^{-1}$ de solo.

A quantidade de $\mathrm{K}$ acumulada na parte aérea aumentou com a elevação
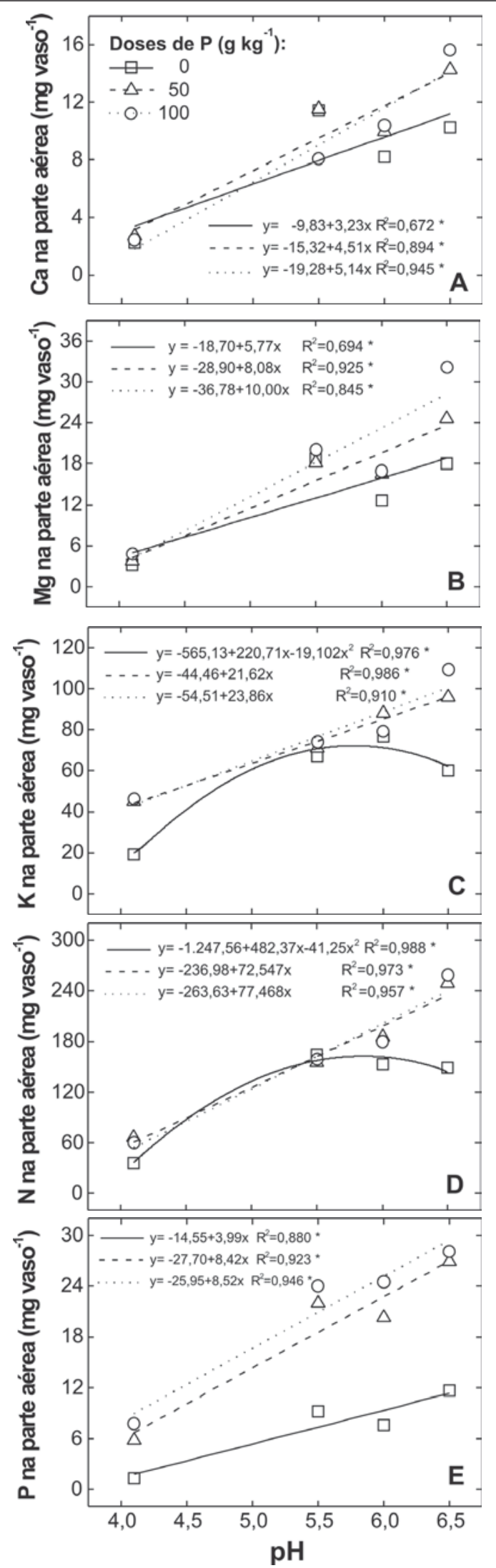

Figura 2. Teor total de $\mathrm{Ca}(\mathrm{A}), \mathrm{Mg}(\mathrm{B}), \mathrm{K}(\mathrm{C}), \mathrm{N}(\mathrm{D})$ e $\mathrm{P}(\mathrm{E})$ na parte aérea de plantas de erva-deSão-João (Hypericum perforatum L.) em função do $\mathrm{pH}$ e da adubação fosfatada (total content of $\mathrm{Ca}(\mathrm{A}), \mathrm{Mg}(\mathrm{B}), \mathrm{K}(\mathrm{C}), \mathrm{N}(\mathrm{D})$ and $\mathrm{P}$ (E) on the aerial part of St. John's Wort plants (Hypericum perforatum $\mathrm{L}$.), on response to $\mathrm{pH}$ and phosphate fertilization). Lages, UDESC, 2003.

*Significativo a $5 \%$ de probabilidade (significant at $5 \%$ probability). 

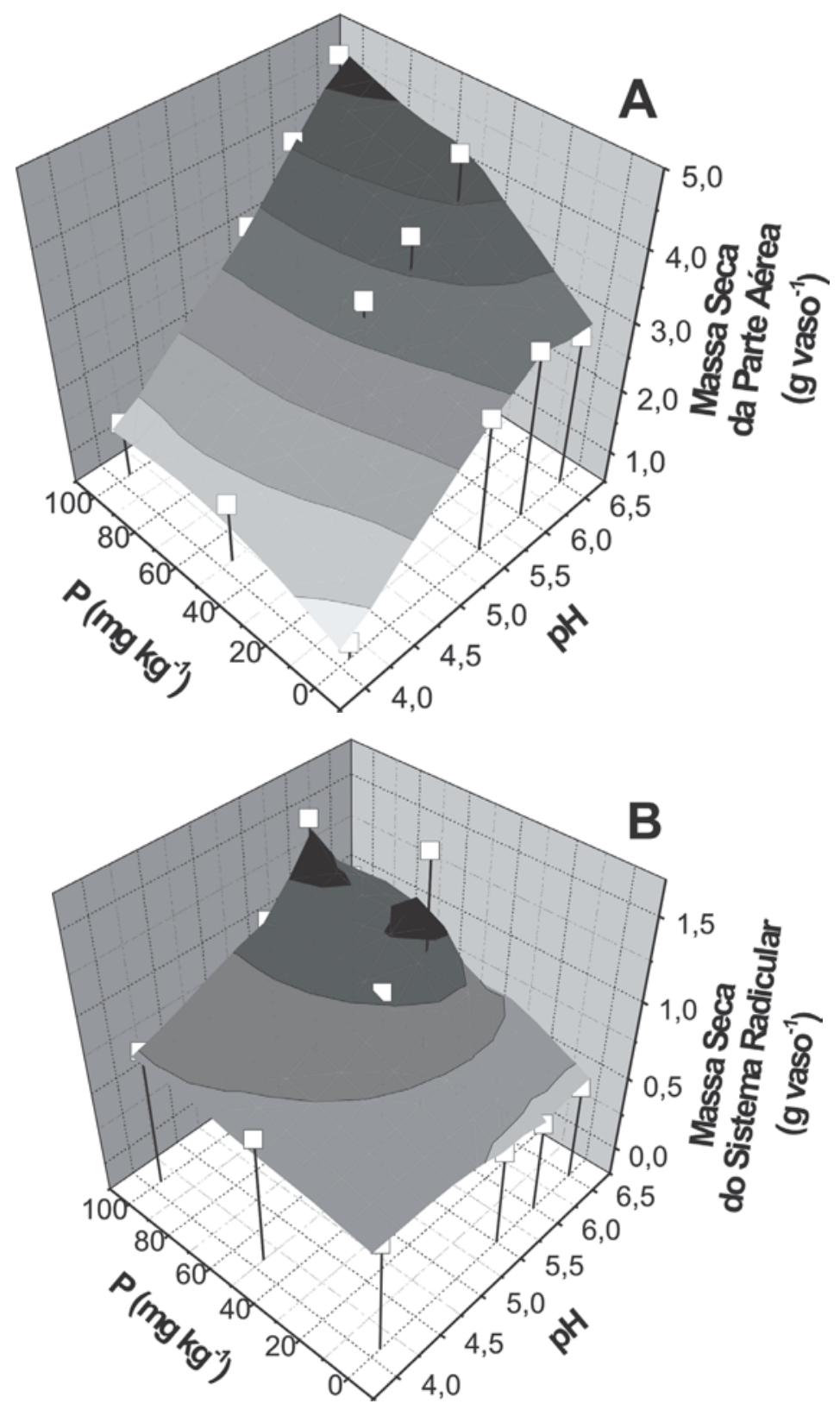

Figura 3. Produção de massa seca da parte aérea (A) e do sistema radicular (B) de erva-deSão-João (Hypericum perforatum L.) em função do $\mathrm{pH}$ e doses de $\mathrm{P}$. (shoot (A) and root (B) dry matter of St. John's Wort plants (Hypericum perforatum L.), in response to $\mathrm{pH}$ and phosphate fertilization). Lages, UDESC, 2003.

do $\mathrm{pH}$, principalmente nos tratamentos que receberam fósforo (Figura 2C). $\mathrm{O}$ teor de $\mathrm{N}$ na parte aérea das plantas seguiu o mesmo comportamento verificado para o K (Figura 2D). A diminuição do acúmulo de $\mathrm{K}$ e $\mathrm{N}$ nos maiores valores de $\mathrm{pH}$, na ausência de $\mathrm{P}$, não afetou, entretanto, a produção de massa seca da parte aérea, uma vez que esta aumentou com a calagem (Figura 3A).

A aplicação de $100 \mathrm{mg} \mathrm{kg}^{-1}$ de P elevou o P no solo mais ácido $(\mathrm{pH} 4,1)$ de
4,84 $\mathrm{mg} \mathrm{kg}^{-1}$ para $18,3 \mathrm{mg} \mathrm{kg}^{-1}$ (incremento de $278 \%$ ), enquanto que nos tratamentos que receberam calagem $(\mathrm{pH}$ $5,5$ a 6,5$)$ o $\mathrm{P}$ passou de $4,19 \mathrm{mg} \mathrm{kg}^{-1}$ para $15,40 \mathrm{mg} \mathrm{kg}^{-1}$ (incremento de 267\%). A adição do calcário provocou diminuição no teor de $\mathrm{P}$ extraível em aproximadamente $15 \%$, e isto provavelmente ocorreu devido à precipitação de $\mathrm{P}$ com $\mathrm{Ca}$, que tem sua concentração aumentada com a calagem (Ernani et al., 2000). Todavia, o acúmulo de $P$ na par- te aérea das plantas aumentou com a elevação do pH (Figura 2E), sendo, na média dos valores, de 4,56 mg vaso ${ }^{-1}$ no $\mathrm{pH} 4,1$, e de 18,$1 ; 17,15$ e $21,74 \mathrm{mg}$ vaso $^{-1}$ nos valores de $\mathrm{pH} 5,5 ; 6,0$ e 6,5, respectivamente.

Houve efeito significativo $(\mathrm{p}<0,05)$ do aumento do $\mathrm{pH}$ e da dose de $\mathrm{P}$ na produção de massa seca da parte aérea e do sistema radicular, sendo que a interação $\mathrm{pH}$ x dose de $\mathrm{P}$ não foi significativa.

A aplicação de calcário e de $\mathrm{P}$ incrementaram a produção de massa seca da parte aérea (Figura 3A). A elevação do $\mathrm{pH}$ do solo para valores iguais ou superiores a 5,5, no tratamento sem aplicação de P, promoveu aumento no rendimento de massa seca de aproximadamente $75 \%$ em relação ao tratamento sem calagem. Este aumento é superior ao causado pela aplicação de fósforo no tratamento sem calagem, independente da dose (50 ou $100 \mathrm{mg} \mathrm{kg}^{-1}$ ), o qual foi de aproximadamente $45 \%$ em relação àquele sem a adição do nutriente (Figura 3A). O maior rendimento de massa seca da parte aérea das plantas de ervade-São-João $\left(4,76 \mathrm{~g} \mathrm{vaso}^{-1}\right)$ ocorreu no tratamento que tinha a combinação do $\mathrm{pH}$ mais elevado $(6,5)$ com a maior dose de $\mathrm{P}$ aplicada (100 $\mathrm{mg} \mathrm{kg}^{-1}$ ), o qual foi $84 \%$ maior que o tratamento testemunha (sem P e sem calcário).

Vários trabalhos reportam os efeitos benéficos da calagem e da adubação com $\mathrm{P}$ na promoção de crescimento de diversas plantas medicinais. Losna (Artemísia annua L.) (Figueira, 1996) e quebra-pedra (Phyllanthus niruri L.) (Becker et al., 2000) apresentaram maior crescimento com a correção da acidez do solo. O efeito da adição de fósforo ao solo no desenvolvimento de plantas medicinais tem sido bastante variável. O P aumentou substancialmente o crescimento vegetativo de pata-devaca (Bauhinia forficata L.) (Ramos, 1999), menta (Kothari et al., 1987) e da erva-de-São-João (Azizi \& Omidbaigi, 2002), porém teve pequeno incremento na camomila (Chamomilla recutita L.) (Corrêa et al., 1991) e na cúrcuma (Curcuma longa L.) (Silva et al., 2004). $\mathrm{A}$ adição desse nutriente não influenciou o desenvolvimento do endro (Anethum graveolens L.) (Lásló, 1979), reduzin- 
do a produção de massa seca do coentro (Coriandrum sativum L.) quando foram utilizadas doses de $\mathrm{P}$ maiores que 112 $\mathrm{kg} \mathrm{ha}^{-1}$ (Oliveira et al., 2004).

O desenvolvimento do sistema radicular da erva-de-São-João aumentou pela aplicação de $\mathrm{P}$ nos tratamentos que receberam calcário, atingindo a máxima produção de massa seca nos tratamentos que tinham $\mathrm{pH}$ 6,0 e que receberam a adição de doses de $\mathrm{P}$ de 50 a $100 \mathrm{mg} \mathrm{kg}^{-1}$ de solo (Figura 3B). Na ausência de $\mathrm{P}$, a adição de calcário reduziu a produção de massa seca do sistema radicular em aproximadamente $22 \%$ em relação ao tratamento sem calagem. Com a correção do $\mathrm{pH}$ para valores acima de 5,8 possivelmente, houve uma redução no teor de P na solução do solo, devido à adição de calcário, que gera um grande aumento na quantidade de $\mathrm{Ca}$ no solo, favorecendo a precipitação do $\mathrm{P}$ na forma de fosfato de cálcio (Ernani et al., 1996). Isto pode ter ocasionado a redução na massa seca do sistema radicular com o incremento no $\mathrm{pH}$ de 6,0 para 6,5 nas doses mais altas de $\mathrm{P}$ (Figura 3B).

Estes resultados mostram que a ervade-São-João responde positivamente à calagem, especialmente com a suplementação adicional de fósforo, através do aumento no acúmulo de $\mathrm{Ca}$, $\mathrm{Mg}, \mathrm{K}, \mathrm{N}$ e P na parte aérea e promoção do crescimento e produção comercial desta espécie.

\section{AGRADECIMENTOS}

Os autores agradecem ao CNPq e à CAPES pelo apoio financeiro a este projeto.

\section{REFERÊNCIAS}

AZIZI M; OMIDBAIGI R. 2002. Effect of NP supply on herb yield, hypericin content and cadmium accumulation of St. John's Wort (Hypericum perforatum L.). Acta Horticulturae 576: 267-271.

BECKER L; FURTINI AE; PINTO JEBP; CARDOSO MG; SANTOS OCD; BARBOSA JM; LAMEIRA OA; SANTIAGO EJA. 2000. Crescimento e produção de alcalóides totais de quebra-pedra em função da calagem e da adubação nitrogenada. Horticultura Brasileira 18: 100-104.

BRISKIN DP; LEROY A; GAWIENOWSKI MC 2000. Influence of nitrogen on the production of hypericins by St. John's Wort. Plant Physiology and Biochemistry 38: 413-420.

COMISSÃO DE FERTILIDADE DO SOLO $\mathrm{RS} / \mathrm{SC}$ (CFS - RS/SC). 2004. Recomendações de adubação e de calagem para os Estados do Rio Grande do Sul e de Santa Catarina. 3. ed. Passo Fundo: SBCS - Núcleo Regional Sul. 394p.

CORRÊA CJ; MING LC; SCHEFFER MC. 1991. Cultivo de plantas medicinais, condimentares e aromáticas. Curitiba: EMATER. $151 \mathrm{p}$.

ERNANI PR; ALMEIDA LA. 1986. Comparação de métodos analíticos para avaliar a necessidade de calcário dos solos de estado de Santa Catarina. Revista Brasileira de Ciência do Solo 10: 143-150.

ERNANI PR; FIGUEIREDO ORA; BECEGATO V; ALMEIDA JA. 1996. Decréscimo na retenção de fósforo no solo pelo aumento do $\mathrm{pH}$. Revista Brasileira de Ciência do Solo 20: 159-162.

ERNANI PR; NASCIMENTO JAL; CAMPOS ML; CAMILO RJ. 2000. Influência da combinação de fósforo e calcário no rendimento de milho. Revista Brasileira de Ciência do Solo 24: 537-544.

FIGUEIRA GM. 1996. Nutrição mineral, produção e teor de artemisinina em Artemisia annua L. Acta Horticulturae 426: 573-577.

KOTHARI SK; SINGH V; SINGH K. 1987. Response of rates and methods of phosphorus application on herb and oil yields and nutrient concentration in Japanese mint (Mentha arvensis L.). Indian Journal of Plant Physiology 108: 691-693.
LÁSLÓ H. 1979. Effect of nutrition supply on yield of dill (Anethum graveolens L.) and essential oil content. Planta Medica 26: 295-296.

LIERES AL von; VOLKMANN B; LIERES AL von. 1994. Relationship between fertilizer, nutrient withdrawal and composition of different medicinal plants in pot experiment. In: CONGRESS OF ALTERNATIVES IN LAND USE AND THE PRODUCTION AND UTILIZATION OF AGRICULTURAL PRODUCTS, 19. Annals... Kassel: VDLUFA. p. 541-544.

OLIVEIRA AP; ARAÚJO LR; MENDES JEMF; DANTAS JÚNIOR OR; SILVA MS. 2004. Resposta do coentro a adubação fosfatada em solo com baixo nível de fósforo. Horticultura Brasileira 22: 87-89.

RAMOS MRC. 1999. Crescimento e nutrição mineral de mudas de pata-de-vaca (Bauhinia forficata L.) em função da aplicação de nitrogênio, fósforo e potássio. Lavras: UFLA. 41 p. (Dissertação mestrado).

SILVA NF; SONNENBERG PE; BORGES JD. 2004. Crescimento e produção de curcuma (Curcuma longa L.) em função de adubação mineral e densidade de plantio. Horticultura Brasileira 22: 61-65.

SOUTHWELL IA; BOURKE CA. 2001. Seasonal variation in hypericin content of Hypericum perforatum. L. Biochemical Systematics and Ecology 56: 437-441.

TAIZ L; ZEIGER, E. 2002. Plant physiology. 3 ed. Sunderland: Sinauer Associates. 690p.

TEDESCO MJ; GIANELLO C; BISSANI CA; BOHNEN H; VOLKWEISS SJ. 1995. Análise do solo, plantas e outros materiais. 2. ed. Porto Alegre: Departamento de Solos UFRGS. 174 p. 